\title{
Estratégias dos estudos métricos da informação para o mapeamento de inovação
}

\author{
Strategies of metric information studies for innovation mapping
}

Mateus Rebouças Nascimento ${ }^{\text {ai }}$

ORCID: https://orcid.org/0000-0001-9211-32

Ana Clara Cândido ${ }^{\text {bii }}$

ORCID: https://orcid.org/0000-0003-1897-3946

Ricardo Augusto Zimmermann ${ }^{\text {ciii }}$

ORCID: https://orcid.org/0000-0002-3398-7134

Patrícia Wielewicki ${ }^{\mathrm{d} i v}$

ORCID: https://orcid.org/0000-0002-9848-8328

Recebido em: 15/07/2021. Aprovado em: 27/08/2021.

Resumo

O presente artigo objetiva identificar estratégias metodológicas existentes no âmbito dos estudos métricos da informação que contribuem para o mapeamento de inovação. Em termos metodológicos, este estudo qualitativo de caráter exploratório e descritivo parte do levantamento da produção de conhecimento relacionada às tipologias dos estudos métricos na base de dados Web of Science, por meio da relação do termo inovação nas palavras-chave e keywords plus dos artigos. Os resultados destacam a aplicação da inovação nas metrias da informação no âmbito da altmetria, bibliometria, cientometria, patentometria e webometria.

Palavras-chave: Inovação. Estratégia. Estudos Métricos da Informação.

\begin{abstract}
This paper aims to identify existing methodological strategies within the metrics studies of information that contribute to the mapping of innovation. In methodological terms, this qualitative exploratory and descriptive study is part of the search for knowledge production related to the typologies of metric studies in the Web of Science database, through the relationship of the term innovation in the keywords and keywords plus of the articles. The results highlight the application of innovation in information metrics in the scope of altmetry, bibliometrics, scientometrics, patentometry and webometrics.
\end{abstract}

Keywords: Innovation. Strategy. Metric Information Studies.

\footnotetext{
${ }^{a}$ Universidade Federal de Santa Catarina. Santa Catarina/Brasil. E-mail: maateusreboucas@gmail.com

${ }^{\mathrm{b}}$ Universidade Federal de Santa Catarina. Santa Catarina/Brasil. E-mail: ana.candido@ufsc.br

${ }^{\mathrm{c}}$ Instituto de Engenharia de Sistemas e Computadores, Tecnologia e Ciência. Porto/Portugal. E-mail: ricardo.a.zimmermann@inesctec.pt

${ }^{\mathrm{d}}$ Universidade do Porto. Porto/Portugal. E-mail: patriciafw@gmail.com 


\section{Introdução}

A aplicação de métodos na Ciência da Informação extrapola a investigação de suas propriedades e fluxos de informação. O caráter multidisciplinar dessa área favorece a análise da constituição de informações de naturezas diversas, o que é feito a partir da utilização de metodologias aplicadas à disseminação, gestão, organização e recuperação da informação em múltiplos contextos.

Em termos científicos, a investigação do conhecimento registrado por meio de canais comunicativos do processo científico, como artigos, capítulos de livros, teses e dissertações, ocorre mediante a aplicação de estudos métricos da informação, compreendido como "métodos e técnicas de mensuração e avaliação quantitativa (estatístico-matemático) da produção, circulação e uso da informação" (ARAÚJO, 2015, p. 44).

A avaliação de caráter quantitativo dos estudos métricos da informação colabora na identificação de como o conhecimento é prospectado, construindo indicadores que destacam os principais atores de determinada área do conhecimento, seus impactos para disciplinas, temáticas discutidas, relações de colaboração entre pesquisadores, universidades e países, registro de patentes, análise de acessos na web, dentre outros indicadores métricos informativos.

Nesse cenário, os estudos métricos permitem mapear estratégias para inovação, compreendendo a inovação como "um processo contínuo que ocorre de forma ordenada e organizada, por isso, depende de um gerenciamento para que ocorra com plenitude" (CÂNDIDO; BERTOTTI; VIANNA, 2017, p. 674). Além de propiciar o desenvolvimento de novos produtos e processos, diversificação, qualidade e absorção de tecnologias aplicadas avançadas (TOMAÉL; ALCARÁ; CHIARA, 2005, p. 99).

A identificação e análise das estratégias utilizadas nos estudos métricos da informação no âmbito da inovação podem contribuir para verificar se o emprego das métricas têm acompanhado as tendências atuais na produção de conhecimento registrado. Nesse sentido, sublinha-se a aplicação da altmetria, bibliometria, cientometria, informetria, webometria e patentometria, essa última voltada para análise de patentes, tipo de propriedade intelectual relacionada diretamente com a inovação.

O objetivo deste artigo é identificar os aportes metodológicos dos estudos métricos da informação com foco no mapeamento de inovação. Para tanto, são analisadas as estratégias que podem ser adotadas em processos inovativos, levantando a produção de conhecimento sobre cada tipologia métrica na base de dados Web of Science. Além disso, 
são apresentadas as relações desses estudos para o campo da inovação e suas aproximações mediante a ocorrência nas palavras-chave e keywords plus dos artigos.

No plano científico, este estudo impacta o domínio da Ciência da Informação à medida que contribui para a construção da analogia dos estudos métricos da informação com o processo de inovação em diferentes áreas do conhecimento. Além disso, favorece a identificação de estratégias métricas que contribuem para o mapeamento e análise da inovação, bem como indicadores de inovação voltados às metrias que compõem esse cenário.

\section{Estudos métricos da informação: conceituações norteadoras}

A comunicação na ciência é compreendida por Garvey (1979) como o conjunto das atividades constituídas na produção, disseminação e uso da informação desde o início do processo de criação científica - da geração de ideias até o aceite e publicação dos resultados. Segundo Mugnaini, Carvalho e Campanatti-Ostiz (2006, p. 316), "para se entender a evolução da ciência, como forma de expressão do conhecimento humano produzido, são utilizadas técnicas de medição".

Nesse processo comunicativo de criação, geração e disseminação de conhecimento, é possível analisar e construir indicadores que prospectam como o conhecimento produzido na ciência está sendo desenvolvido, por meio dos estudos métricos da informação, culminando como subsídios para áreas do conhecimento, na medida em que se aproximam, oferecendo conceitos, metodologias, procedimentos e recursos (OLIVEIRA, 2018, p. 33).

Noronha e Maricato (2008, p. 122) ressaltam como os estudos métricos podem ser aplicados no âmbito do conhecimento na:

\footnotetext{
[...] dinâmica e a participação da C\&T em escala internacional (através da comparação entre dois ou mais países), nacional (entre dois ou mais estados), local (entre instituições de uma mesma cidade ou região).Cada uma dessas categorias de análise podem ser subdividida e aprofundada, surgindo novas variáveis e abordagens, por campo de atuação (linhas de pesquisa), por pesquisadores (formação, titulação), por colaboração (trabalhos em co-autoria, sociabilidade entre autores), assuntos, tipos documentais (periódicos, teses, dissertações, eventos, etc.), instituições (universidades, centros de pesquisa, empresas), departamentos, cursos, disciplinas, etc.
}

Compreendendo que os estudos métricos são "reflexos atuais da atividade da informação científica/tecnológica/empresarial e de seus sistemas de controle, como as estratificações científicas" (STANCATTI; PINTO, 2021, p. 6), existem subcampos dentro dos estudos métricos da informação que aplicam métricas em tipos de objetos distintos 
como a altmetria, arquivometria, bibliometria, cibermetria, cientometria, informetria, patentometria e webometria, apresentadas no quadro abaixo as suas aplicações conforme a visão de teóricos da área.

\begin{tabular}{|c|c|}
\hline $\begin{array}{l}\text { Métrica da } \\
\text { Informação }\end{array}$ & Aplicação \\
\hline Altmetria & $\begin{array}{l}\text { Novo grupo de métricas baseadas em eventos de mídia social relacionados com a } \\
\text { comunicação científica (BORBA; MARINHO; CAREGNATO, 2017). }\end{array}$ \\
\hline Arquivometria & $\begin{array}{l}\text { O objeto de estudo é o fundo documentário e seus usuários, contando com variáveis } \\
\text { da estrutura do Arquivo (ações e gestão) e circulação de consultas, abordando } \\
\text { métodos de frequências e distribuição (PINTO, 2011). }\end{array}$ \\
\hline Bibliometria & $\begin{array}{l}\text { Produção e uso de documentos, organização de serviços bibliográficos, documentos } \\
\text { (livros, artigos, teses), autores, usuários (NORONHA; MARICATO, 2008). }\end{array}$ \\
\hline Cibermetria & $\begin{array}{l}\text { Internet, ciberespaço como chats, mailing lists, grupos de discussão, muds e a } \\
\text { WWW (VANTI, 2005) }\end{array}$ \\
\hline Cientometria & $\begin{array}{l}\text { Estudo dos aspectos quantitativos da ciência enquanto uma disciplina ou atividade } \\
\text { econômica (MACIAS-CHAPULA, 1998). }\end{array}$ \\
\hline Informetria & $\begin{array}{l}\text { Estudo dos aspectos quantitativos da informação em qualquer formato, e não apenas } \\
\text { registros catalográficos ou bibliografias, referente a qualquer grupo social, e não } \\
\text { apenas aos cientistas (MACIAS-CHAPULA, 1998). }\end{array}$ \\
\hline Patentometria & $\begin{array}{l}\text { Conhecer atividades tecnológicas e inovadoras de países, áreas e instituições } \\
\text { (NORONHA; MARICATO, 2008). }\end{array}$ \\
\hline Webometria & $\begin{array}{l}\text { Estudo dos aspectos quantitativos da construção e uso dos recursos de informação, } \\
\text { estruturas e tecnologias da Web a partir de abordagens (SANTOS; } \\
\text { ALBUQUERQUE, 2017). }\end{array}$ \\
\hline
\end{tabular}

Quadro 1 - Tipos de estudos métricos e suas aplicações Fonte: Elaborado pelos autores (2021).

As tipologias dos estudos métricos da informação são inter-relacionadas na perspectiva de diversos autores (BJÖRNEBORN; INGWERSEN, 2004; PINTO, 2011; GOUVEIA, 2013; SANZ-CASADO; GARCIA-ZORITA, 2014; ARAÚJO, 2015) como destacado por Curty e Delbianco (2020), com semelhanças e particularidades entre modelos destacadas em formato de elipses. Após a análise dos modelos citados, Curty e Delbianco (2020, p. 16) apresentam a representação das diferentes metrias por meio de três dimensões: espaço (locus), foco (focus) e fluxo (fuxus) - (Figura 1). 


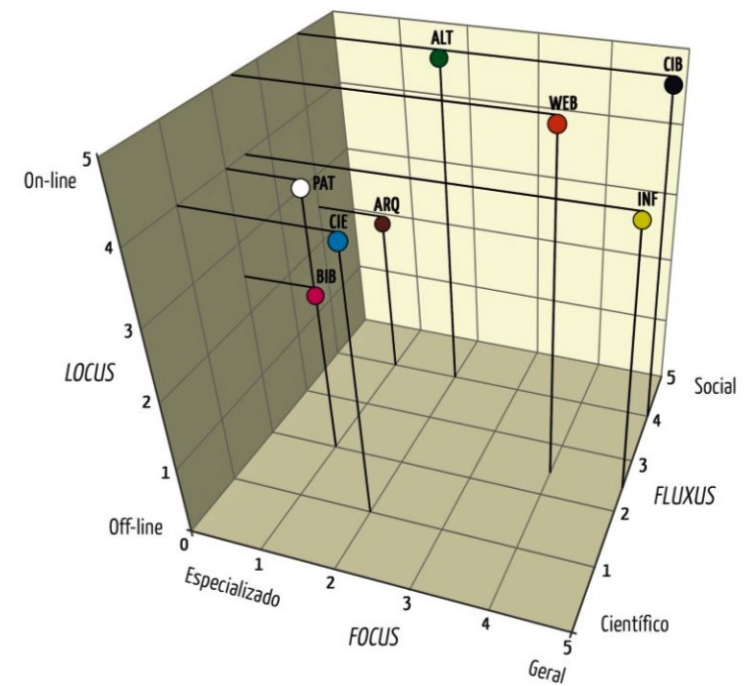

Figura 1 - Abordagens dos estudos métricos e suas dimensões Fonte: Curty e Delbianco (2020, p. 16).

A primeira dimensão (locus) destaca o ambiente no qual as métricas são capturadas, mapeando em universos on-line e off-line. Segundo as autoras, infere-se que a arquivometria e bibliometria geram métricas nos dois universos, enquanto a patentometria, informetria e a cientometria aplicam no universo on-line, todavia, a altmetria, cibermetria e webometria possuem foco total no ambiente on-line na análise dos dados de pesquisa.

Quanto ao focus de aplicação das tipologias dos estudos métricos da informação, as autoras pontuam que a arquivometria, bibliometria e patentometria são norteadoras em práticas informacionais, destacando que "aplicação genérica e metonímica da Bibliometria não coaduna com os desdobramentos métricos resultantes dos avanços conceituais e de aplicação dos EMI” (CURTY; DELBIANCO, 2020, p. 16).

A circulação desses subcampos, pontuada na última dimensão (fluxus), são explicitados na cientometria voltada para o fluxo científico, a bibliometria e patentometria em seguida, avaliando e mensurando métricas na ciência, pelos quais as autoras reforçam a infometria e webometria como posição central no eixo, tendo em vista seus tipos de estudo em âmbito científico e social, perpassando nos dois contextos.

Com isso, considerando a natureza interdisciplinar e a constante transformação das tipologias de estudos métricos, consoante as múltiplas formas de comunicar ciência (SANTOS; ALBUQUERQUE, 2017, p. 9), é possível verificar como a inovação acontece nesse meio. Para tanto, entende-se que os indicadores métricos favoreçam o mapeamento de inovação em diversos pretextos, no âmbito de produtos e serviços. 


\section{Inovação: perspectivas teórico-conceituais}

Sáenz e Capote (2002, p. 69) contextualizam o processo de inovação como "a integração de conhecimentos novos e de outros existentes para criar produtos, processos, sistemas ou serviços novos ou melhorados". Na perspectiva do conceito básico de inovação, o Manual de Oslo (OECD, 2018, p. 32, tradução nossa) aponta a atividade como "um produto ou processo novo ou aprimorado (ou combinação dos mesmos) que difere significativamente dos produtos ou processos anteriores da unidade e que foi disponibilizado para usuários potenciais (produto) ou colocado em uso pela unidade (processo)".

Nesse sentido, o conhecimento norteia a inovação aplicada a cenários distintos; Souza Neto, Orlandi e Santos (2014, p. 520) destacam o foco da inovação aplicada em serviços nos "investimentos no desempenho dos empregados, inovação operacional contínua, inovação estratégica contínua, modelo de negócios que facilite a escalabilidade”. Assim, sublinha-se a importância do uso de estratégias aplicadas no âmbito da inovação, constituído na composição de serviços inovadores para sociedade utilizar em seu cotidiano.

No âmbito da relação entre inovação e academia, Audy (2017, p. 76) sugere que ela "envolve a criação de novos projetos, conceitos, formas de fazer as coisas, sua exploração comercial ou aplicação social e a consequente difusão para o restante da economia ou sociedade". Assim, essa interação desenvolve-se na interface entre empresas, universidades, centros de pesquisa, espaços propiciadores de inovação e envolve a gestão da propriedade intelectual.

Todavia, para propor a inovação em produtos e serviços, existe a necessidade de apoio financeiro oriundo de fontes diversas. No Brasil, a título de exemplo, destacam-se três tipos de fomento público: fundos setoriais, subvenção econômica e o Sistema Brasileiro de Tecnologia (SIBRATEC). Os fundos setoriais colaboram no fortalecimento do Sistema de Ciência e Tecnologia (C\&T), ofertando editais que visam à inovação, mediados pelo Ministério de Ciência, Tecnologia e Inovação (MCTI).

Por meio da Lei de Inovação $n^{0} 10.973$ (BRASIL, 2004), o marco legal brasileiro concretizou-se mediante a essa prescrição, permitindo o uso de recursos públicos não reembolsáveis diretamente em empresas no âmbito público e privado com foco no desenvolvimento de produtos inovativos para o país, contribuindo assim para práticas inovativas. 
O SIBRATEC, criado mediante ao Decreto $\mathrm{n}^{0}$ 6.259, de 21 de novembro de 2007, apoia empresas que utilizam a inovação em suas práticas. Esse mecanismo oferece suporte no desenvolvimento tecnológico de empresas brasileiras, propiciando e incentivando o mercado para propor inovações em produtos e serviços.

Nesse sentido, este estudo defende a relevância de iniciativas de mapeamento das estratégias de inovação com vistas à oferta de recursos para essas práticas na sociedade. As dimensões aplicadas nos estudos métricos da informação são sinalizadas como um recurso de pesquisa capaz de contribuir para potencializar a inovação.

\section{Procedimentos metodológicos}

Do ponto de vista metodológico, a pesquisa pode ser considerada do tipo exploratório e descritivo, uma vez que tem como objetivo proporcionar maior aproximação com o problema, com vistas a torná-lo mais explícito ou a construir hipóteses (GIL, 2002, p. 41). O estudo pretende, ainda, descrever com exatidão os fatos e fenômenos aplicáveis (FIGUEIREDO, 2008, p. 94). Quanto à abordagem do problema, trata-se de um estudo qualitativo, pois busca compreender a relação dos estudos métricos da informação com a inovação, mediante os estudos desenvolvidos no campo por meio da análise de conteúdo.

Para identificar a relação entre estes subcampos, foi realizado um levantamento dos artigos voltados à temática de estudos métricos da informação com a inovação na base de dados Web of Science (WOS) em junho de 2021, por meio de uma estratégia de busca sistematizada (Quadro 2). Foi utilizada como filtro de pesquisa a ocorrência dos termos no título, resumo e palavras-chave, utilizando as principais métricas informativas relacionadas ao termo inovação: altmetrics, archivometrics, bibliometrics, cybermetrics, scientometrics, informetrics, patentometrics e webometrics.

No levantamento inicial foram totalizados 470 artigos, posteriormente submetidos a uma etapa de refinamento. Nessa seleção foram considerados os artigos que possuíam o tipo de estudo métrico e o termo inovação nas palavras-chave do autor e keywords plus, de modo a identificar os documentos que destacam a relação direta para o mapeamento de inovação. Esse processo culminou na seleção e análise de 272 documentos. 


\begin{tabular}{|c|c|c|c|}
\hline Métrica da Informação & Estratégia de Busca & $\begin{array}{l}\text { Artigos } \\
\text { Recuperados }\end{array}$ & $\begin{array}{l}\text { Artigos } \\
\text { considerados }\end{array}$ \\
\hline Altmetria & "Altmetrics" AND "Innovation" & 14 & 7 \\
\hline Arquivometria & "Archivometrics" AND "Innovation" & 0 & 0 \\
\hline Bibliometria & "Bibliometrics" AND "Innovation" & 345 & 211 \\
\hline Cibermetria & "Cybermetrics" AND "Innovation" & 0 & 0 \\
\hline Cientometria & "Scientometrics" AND "Innovation" & 99 & 44 \\
\hline Informetria & "Infometrics" AND "Innovation" & 1 & 0 \\
\hline Patentometria & "Patentometrics" AND "Innovation" & 12 & 6 \\
\hline Webometria & "Webometrics" AND "Innovation" & 9 & 4 \\
\hline
\end{tabular}

Com isso, foram extraídos ${ }^{\mathrm{e}}$ os registros completos e referências citadas desses documentos na WOS, no formato separado por tabulações, para posteriormente a aplicação em um software métrico. Os termos arquivometria, informetria e cibermetria não tiveram artigos recuperados em relação à inovação. A escolha da WOS como base de dados para o mapeamento desta pesquisa justifica-se pelo seu impacto e relevância em várias disciplinas acadêmicas, permitindo acesso a diferentes bancos de dados que fazem referência à pesquisa interdisciplinar (CLARIVATE ANALYTICS, 2017).

Para geração dos grafos de análise das temáticas principais que formam a relação da inovação com os estudos métricos da informação, utilizou-se o software métrico VOSViewer ${ }^{\mathrm{f}}$ na versão 1.6.16. A composição da rede de conhecimento teve como base as palavras-chave e keywords plus dos artigos, considerando o mínimo de 5 ocorrências do termo para compor o mapa, utilizando o layout networking visualization para visualização dos resultados, compreendendo que na rede, os itens são representados por círculos e o tamanho significa o determinado peso da temática, quanto maior o peso, maior é o círculo (VAN ECK; WALTMAN, 2018, p. 8).

\footnotetext{
${ }^{\text {e }}$ Como extrair a coleta de dados na Web of Science para aplicação em análises de estudos métricos da informação: https://www.youtube.com/watch?v=dMSKFVwx Uo

${ }^{\mathrm{f}} \mathrm{O}$ software VOSviewer pode ser instalado gratuitamente disponível para Microsoft Windows, MAC Os X e outros sistemas: https://www.vosviewer.com/download
} 
A relação entre as estratégias dos estudos métricos para o mapeamento de inovação foi analisada com base em estudos pautados nos tipos de metrias da informação. A partir dos 272 artigos analisados observou-se que as dimensões dos estudos altmétricos, bibliométricos, cientométricos, patentométricos e webométricos contribuem para mensurar a produção de: (i) conhecimento sobre a inovação em bases de dados, (ii) uso de métricas alternativas, (iii) as disciplinas transversais ao contexto de estudo; (iv) o investimento econômico no âmbito da inovação; (v) registro de patentes e de invenções (mediante ao uso de indicadores contextualizados na patentometria). O Quadro 3 apresenta as estratégias cuja aplicação foi identificada no âmbito da bibliometria e da cientometria para o mapeamento de inovação.

\begin{tabular}{|c|c|c|}
\hline $\begin{array}{l}\text { Métrica da } \\
\text { Informação }\end{array}$ & Estratégias & Estudos aplicados \\
\hline \multirow{5}{*}{$\begin{array}{l}\text { Bibliometria e } \\
\text { Cientometria }\end{array}$} & $\begin{array}{l}\text { Mapeamento das principais regiões e tópicos de } \\
\text { tendências em pesquisa de políticas de inovação. } \\
\text { Analisa a estrutura intelectual do domínio, os desafios } \\
\text { estratégicos, o desenvolvimento e a aplicação } \\
\text { industrial }\end{array}$ & $\begin{array}{l}\text { Lopez-Rubio,Roing-Tierno \& } \\
\text { Mas-Tur (2021); D'Angelo \& } \\
\text { Magnusson (2021) }\end{array}$ \\
\hline & $\begin{array}{l}\text { Análise cientométrica de publicações em diferentes } \\
\text { áreas do conhecimento visando avaliar as pesquisas e } \\
\text { potencialidades, assim como identificar o nível de } \\
\text { inovação por meio dos produtos mencionados nos } \\
\text { artigos }\end{array}$ & $\begin{array}{l}\text { Masara; Van der Poll \& Maaza } \\
(2021)\end{array}$ \\
\hline & $\begin{array}{l}\text { Identificação dos grupos de pesquisa mais atuantes } \\
\text { em inovação. Mapeia os componentes importantes } \\
\text { para potencialidades do grupo }\end{array}$ & $\begin{array}{l}\text { Forouldi; Akarsu,Marvi \& } \\
\text { Balakrishnan (2021) }\end{array}$ \\
\hline & $\begin{array}{l}\text { Avaliação da evolução e perspectivas dos sistemas de } \\
\text { inovação. Mapeia os principais atores que o } \\
\text { constituem }\end{array}$ & $\begin{array}{l}\text { Lopez-Rubio, Roing-Tierno \& } \\
\text { Mas-Verdu (2021) }\end{array}$ \\
\hline & $\begin{array}{l}\text { Análise de periódicos que publicam sobre inovação. } \\
\text { Identifica o panorama temático, compreendendo a } \\
\text { influência das publicações da revista e caminhos } \\
\text { inovativos futuros }\end{array}$ & Rose; Holzle \& Bjork (2020) \\
\hline
\end{tabular}

As estratégias dos estudos bibliométricos e cientométricos prospectam por meio da análise de cocitações, da identificação das origens de processos inovativos em artigos científicos, além do acoplamento bibliográfico. Esses elementos permitem a visualização abrangente do status de pesquisa sobre inovação, pontuando também como estratégia a identificação da colaboração em sistemas de inovação. Isso coloca em evidência os principais atores inovativos (instituições e países) mediante a perspectiva em análise de 
redes sociais, além de destacar os respectivos graus de influência no sistema e medir o impacto dos periódicos e suas comunicações sobre inovação.

O Quadro 4 apresenta as dimensões do mapeamento de inovação na aplicação de métricas alternativas e contexto da web. No âmbito da utilização da altmetria observou-se o foco nas hélices da inovação em redes sociais, com vistas ao mapeamento dos resultados de inovação em rede na perspectiva do conhecimento, inovação, consenso e governo estudo de avaliação do desempenho de múltiplos grupos de pessoas que formam ecossistemas de inovação desenvolvidos por Moradi e Dohkani (2020).

\begin{tabular}{|c|c|c|}
\hline $\begin{array}{l}\text { Métrica da } \\
\text { Informação }\end{array}$ & Estratégias & Estudos aplicados \\
\hline \multirow[t]{2}{*}{ Altmetria } & $\begin{array}{l}\text { Avaliação do desempenho de indivíduos, } \\
\text { organizações, instituições e países nas redes sociais } \\
\text { focadas para inovaça,o, por meio do modelo de Hélice } \\
\text { Quádrupla examinando os resultados da inovação, } \\
\text { conhecimento, consenso e governo nas redes. }\end{array}$ & Moradi e Dohkani (2020) \\
\hline & $\begin{array}{l}\text { Análise de formas de inovação em espaços abertos } \\
\text { por meio do impacto em redes sociais. }\end{array}$ & Frias e Garcia (2017) \\
\hline \multirow{2}{*}{ Webometria } & $\begin{array}{l}\text { Mapeamento na web para avaliação de menções e } \\
\text { redes de hiperlinks com foco em práticas de inovação, } \\
\text { indicando interações entre atores, número de acessos, } \\
\text { órgãos inovativos. }\end{array}$ & Kim e Park (2014) \\
\hline & $\begin{array}{l}\text { Análise de links para investigação de apoio a P\&D em } \\
\text { inovação por meio da webometria, identificando redes } \\
\text { de setores industriais, universidades e órgãos públicos } \\
\text { que fomentam o desenvolvimento de inovação em } \\
\text { parques científicos. }\end{array}$ & Minguillo e Thelwall (2012) \\
\hline
\end{tabular}

Quadro 4 - Dimensões na altmetria e webometria para o mapeamento de inovação Fonte: Dados da pesquisa (2021).

No contexto da web, o mapeamento de inovação culmina na análise de menções e acesso em hiperlinks textuais de sites, com destaque para a visualização em órgãos e instituições. Essa situação permite a identificação dos principais atores organizacionais que propiciam o financiamento e apoio a P\&D em inovação mediante ao uso de metodologias webométricas. A análise é realizada por meio do número de acessos, o quantitativo de páginas recuperadas em mecanismos de busca que comunicam processos inovativos, além dos downloads que são analisados, propiciando indicadores que inferem o impacto de determinado documento. 


\begin{tabular}{|c|c|c|}
\hline $\begin{array}{l}\text { Métrica da } \\
\text { Informação }\end{array}$ & Estratégias & Estudos aplicados \\
\hline \multirow{4}{*}{ Patentometria } & $\begin{array}{l}\text { Análise sistemática de tecnologias emergentes à } \\
\text { inovação por meio de banco de dados de patentes. }\end{array}$ & Li; Pak e Bi (2019) \\
\hline & $\begin{array}{l}\text { Análise de patentes concedidas a instituições } \\
\text { registradas em banco de dados de patentes. Aponta as } \\
\text { tendências de processos e produtos de inovação } \\
\text { tecnológica. }\end{array}$ & Hurtado (2018) \\
\hline & $\begin{array}{l}\text { Mapeamento de pedidos de patentes ou modelo de } \\
\text { utilidade para levantar as tecnologias de processos e } \\
\text { produtos inovativos. }\end{array}$ & $\begin{array}{l}\text { Fagundes, Ferreira, Motta e } \\
\text { Leal (2016) }\end{array}$ \\
\hline & $\begin{array}{l}\text { Construção de indicadores patentométricos que } \\
\text { apresentam estatísticas de patentes. }\end{array}$ & $\begin{array}{l}\text { Watanabe, Tsuhi e Brown } \\
\text { (2001) }\end{array}$ \\
\hline
\end{tabular}

Quadro 5 - Dimensões na patentometria para o mapeamento de inovação Fonte: Dados da pesquisa (2021).

O emprego da patentometria para o mapeamento de inovação consiste na construção de indicadores que perpassam a análise de patentes concedidas e registradas, além da avaliação da velocidade de comercialização e o esforço de comercialização por parte das organizações responsáveis. São explorados dados sobre inovações mediante ao registro de patentes em bancos de dados (SILVA et al., 2018) para analisar, por exemplo: (i) o volume de produtos tecnológicos, processos e técnicas desenvolvidos, (ii) a internacionalização de pesquisas na relação de publicações em revistas internacionais, (iii) as patentes internacionais, dentre outras demandas que podem ser analisadas no mapeamento de inovação.

Quanto às facetas e temáticas norteadoras dos estudos métricos focadas na inovação, a Figura 2 (gerada no software VOSViewer) apresenta, por meio da análise de concorrência de palavras-chave e keywords plus dos artigos analisados, quais são os conhecimentos inerentes à aplicação estratégica para inovação. Foi identificado um total de 118 vertentes que constituem o mapa e incluem: a aplicação da inovação na ciência e seus impactos, a gestão e o conhecimento produzido para inovação, além da tecnologia e o empreendedorismo permeando esses aspectos. 


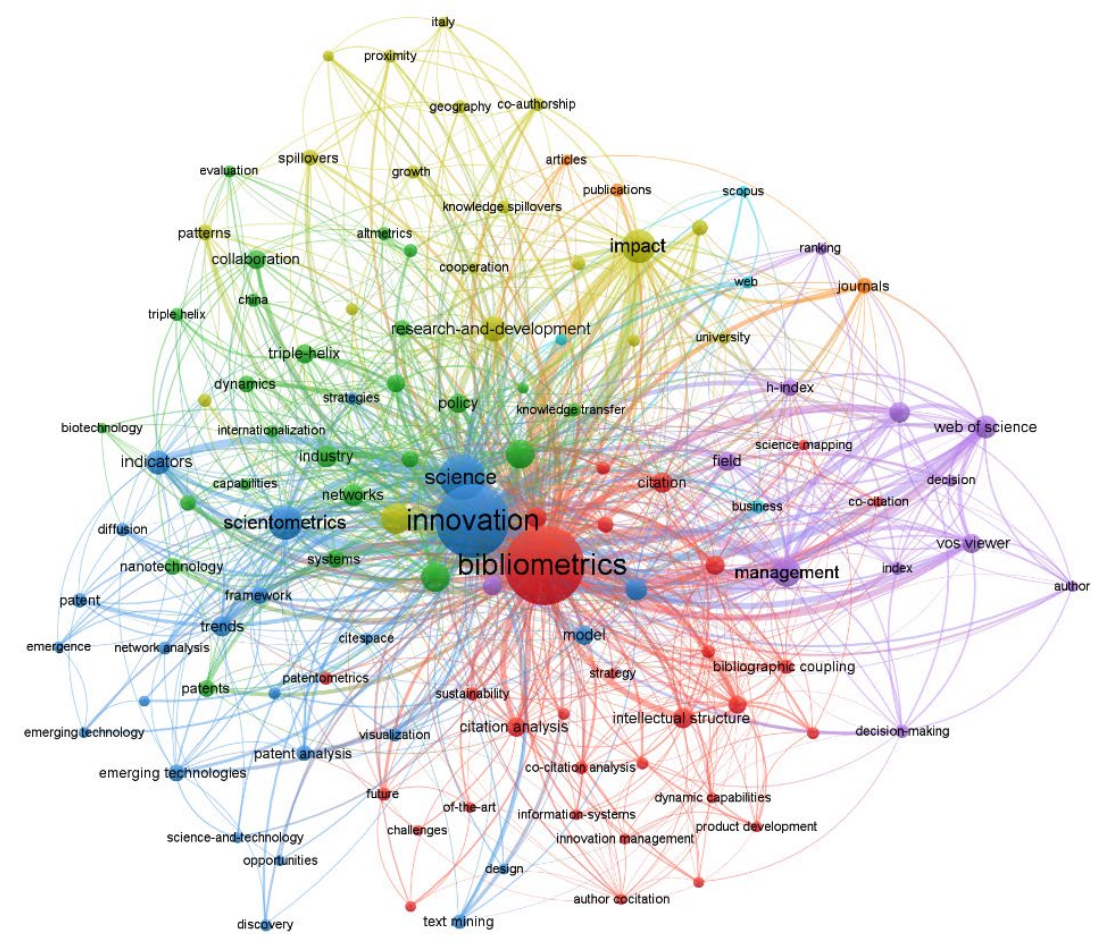

象 vosviewer

Figura 2 - Mapa de conhecimentos dos estudos métricos focados para inovação Fonte: Dados da pesquisa (2021).

Destaca-se como temática principal o termo bibliometria, apresentado no centro do mapa com 204 ocorrências e 1.064 conexões com outras vertentes. Identificou-se que muitos estudos bibliométricos mensuram a produção de conhecimento sobre a inovação (178 ocorrências e 968 conexões). Analisando os dados por meio da coloração dos clusters - definidos a partir das aproximações da inovação com as temáticas - destaca-se que a bibliometria (tonalidade vermelha) é pautada no mapeamento da estrutura intelectual, estratégias, acoplamento bibliográfico, análise de cocitação, dinâmicas, desenvolvimento de produto, gestão da inovação, desafios, modelos de negócios, inovação aberta e empreendedorismo.

A análise da relação entre o cluster vermelho e o cluster roxo, demonstra que ambos são relacionados a escolhas metodológicas dos estudos bibliométricos como: (i) a base de dados para coleta (WOS e Scopus) (ii) os campos a serem extraídos (autores, títulos, periódicos); e (iii) os softwares utilizados para geração dos grafos (vosviewer).

Quanto à inovação, as temáticas emergentes estão tonalizadas de azul, possuindo relação direta com a cientometria e a geração de indicadores, pontuada na análise de tendências, ciência e tecnologia, mineração de texto para o mapeamento de inovação, além de patentes e modelos inovativos. Nesse sentido, a cientometria corrobora para o uso de frameworks, citespace e previsão de tecnologias emergentes, por meio do estudo de 
disciplinas da ciência e aspectos de P\&D. A cientometria, portanto, é particularmente relevante para análises que prospectam o desenvolvimento da ciência em áreas do conhecimento que são inerentes à inovação, como a Ciência da Informação, Ciência da Computação, Engenharias, dentre outras.

Os indicadores mapeados na análise do cluster apresentados na cor azul culminam em: avaliação, colaboração, identificação de capacidades, tendências, análise de redes de inovação, tecnologias emergentes, patentes, difusão e impacto e spillovers. Esse último é pautado na difusão do conhecimento para aplicação da inovação, de forma a que um conhecimento deixe de ser exclusividade de uma empresa e que o acesso seja disponibilizado a outros, contribuindo para a consolidação do conhecimento coletivo. Esse processo contribui para a superação de barreiras à inovação em empresas menores - como altos custos para obter informações críticas, dimensionados em aspectos gerenciais, estratégicos e relacionais (VILELA JUNIOR, 2010).

\section{Considerações Finais}

O objetivo desse artigo foi identificar estratégias metodológicas sistematizadas existentes no âmbito dos estudos métricos da informação que contribuem para o mapeamento de inovação. A partir das análises realizadas, pode-se inferir que os estudos métricos da informação colaboram para o mapeamento da inovação, uma vez que favorecem a identificação dos atores principais no domínio científico que investigam temáticas inovativas por meio de técnicas bibliométricas. Também foram analisados os indicadores científicos voltados para análise da produtividade, como os tipos de documentos publicados, colaboração com outros atores na análise de redes sociais, assim como internacionalização das práticas de inovação em suas produções intelectuais.

Conclui-se que as métricas da informação permeiam a análise de inovação em múltiplos aspectos, como a cientometria pontuada para estudos de disciplinas na ciência contextualizando o desenvolvimento econômico, propiciando estratégias que identifiquem o fomento para inovação e a importância de P\&D em inovação para disciplinas em diversas áreas do conhecimento. Destaca-se que a webometria corrobora na análise da inovação no contexto da web no estudo de links e do impacto de iniciativas inovadoras.

Neste contexto, a patentometria torna-se o elo norteador estratégico de análise da inovação no meio da propriedade intelectual, no sentido de que as patentes são invenções que se utilizam de preceitos inovativos para sua consolidação, em um processo que ocorre seguindo critérios estabelecidos para definir se a criação é algo inovador para sociedade. A 
análise explicita quais países mais prospectam a inovação em documentos de patentes, empresas e universidades que possuem maior quantitativo de registros, áreas do conhecimento nos quais os registros de patentes de inovação acontecem, dentre outros indicadores patentométricos.

Para trabalhos futuros, sugere-se a construção de indicadores de inovação baseados nos estudos métricos da informação para cada metria, analisando a necessidade de propor a consolidação da relação das métricas com a inovação. Sugere-se uma análise mediante a definição de quais indicadores podem ser gerados com viés inovativo a colaborar com agências de fomento para financiamento de práticas inovativas, assim como na construção de ecossistemas de inovação da produção de conhecimento sobre inovação na ciência.

\section{Referências}

ARAUJO, R. F. de. Estudos métricos da informação na web e o papel dos profisssionais da informação. Bibl. Univ., Belo Horizonte, v. 2, n. esp., p. 42-65, 2015. Disponível em: https://periodicos.ufmg.br/index.php/revistarbu/article/view/3074. Acesso em: 20 maio. 2021.

AUDY, J. A inovação, o desenvolvimento e o papel da Universidade. Estudos Avançados, v. 31, n. 90, p. 75-87, 2017. Disponível em: http://www.scielo.br/pdf/ea/v31n90/0103-4014-ea-31-90-0075.pdf. Acesso em: 19 maio. 2021.

BJÖRNEBORN, L.; INGWERSEN, P. Toward a basic framework for webometrics. Journal of the American Society for Information Science and Technology, Nova York, v. 55, n. 14, p. 1216-1227, 2004. Disponível em:

https://onlinelibrary.wiley.com/doi/abs/10.1002/asi.20077. Acesso em: 10 maio. 2021.

BORBA, V. R.; MARINHO, A. C. M.; CAREGNATO, S. E. Análise do termo "Repositório Institucional" no Twitter: um estudo altmétrico. Em Questão, Porto Alegre, ed. esp. 5 EBBC, v. 23, p. 290-308. 2017. Disponível em:

https://seer.ufrgs.br/index.php/EmQuestao/article/view/68086. Acesso em: 10 maio. 2021.

BRASIL. Lei n. 10.973, de 02 de dezembro de 2004. Dispõe sobre incentivos à inovação e à pesquisa científica e tecnológica no ambiente produtivo e dá outras providências. Diário Oficial da República Federativa do Brasil, Brasília, 03 dez. 2004.

CÂNDIDO, A. C.; BERTOTTI, P. S. da S.; VIANNA, W. B. Análise de Redes Sociais das temáticas: Gestão da Informação e da Inovação. RDBCI, Campinas, v. 15, n. 3, p. 670-692, 2017. Disponível em:

https://periodicos.sbu.unicamp.br/ojs/index.php/rdbci/article/view/8649761. Acesso em: 26 maio. 2021. 
CLARIVATE ANALYTICS. Web of Science. 2017. Disponível em: http://wilearncap.asuscomm.com:81/wikipedia_en_all_novid_201708/A/Web_of_Science. html. Acesso em: 15 jun. 2021.

CURTY, R. G.; DELBIANCO, N. R. As diferentes metrias dos estudos métricos da informação: evolução epistemológica, inter-relações e representações. Encontros Bibli, v. 25, 2020. DOI https://doi.org/10.5007/1518-2924.2020.e74593.

D'ANGELO, V.; MAGNUSSON, M. A Bibliometric Map of Intellectual Communities in Frugal Innovation Literature. IEE Transactions on Engineering Management, v. 68, n. 3, 2021. Disponível em: https://ieeexplore.ieee.org/document/9102408/. Acesso em: 20 jun. 2021.

FAGUNDES, M. C.; FERREIRA, M. D.; MOTTA, G. D.; LEAL, I. C. Patentometric analysis of the process Technologies of companhia siderurgica nacional (CSN). Revista Geintec Gestão Inovação e Tecnologias, v. 6, n. 2, p. 43-47, 2016. Disponível em: http://www.iaabr.com/journals/jaefa/jaefa-library/jaefa-vol-5-number-1-2015/. Acesso em: 20 jun. 2021.

FIGUEIREDO, N. M. A. (Org.). Método e metodologia na pesquisa científica. 3. ed. São Caetano do Sul: Yendis Editora, 2008.

FOROUDI, P.; ARKASU, T. N.; MARVI, R.; BALAKRISHNAN, J. Intellectual evolution of social innovation: a bibliometric analysis and avenues for future research trends. Industrial Marketing Management, v. 93, p. 446-465, 2021. Disponível em: https://www.sciencedirect.com/science/article/abs/pii/S0019850119306340. Acesso em: 20 jun. 2021.

FRIAS, E. R.; GARCIA, N. R. Social Labs in Universities: Innovation and impact in Medialab UGR. Comunicar, v. 25, n. 51, p. 29-38, 2017. Disponível em: https://doi.org/10.3916/C51-2017-03. Acesso em: 20 jun. 2021.

GARVEY, W. D. Communication: the essence of science. Oxford: Pergamon Press, 1979.

GIL, A. C. Como elaborar projeto de pesquisa. 4. ed. São Paulo: Atlas, 2002.

GOUVEIA, F. C. Altmetria: métricas de produção científica para além das citações. Liinc em Revista, Rio de Janeiro, v. 9, n. 1, p. 214-227, maio. 2013. Disponível em: http://revista.ibict.br/liinc/article/view/3434. Acesso em: 10 maio. 2021.

HURTADO, R. E. G. Patent analysis of Colombian technologic al innovation 1991-2013. Investigacion Bibliotecologica, v. 32, n. 77, p. 133-150, 2018. Disponível em: https://doi.org/10.22201/iibi.24488321xe.2018.77.57859. Acesso em: 20 jun. 2021.

KIM, J. H.; PARK, H. W. Food policy in cyberspace: A webometric analysis of national food clusters in South Korea. Government Information Quarterly, v. 31, n. 3, p. 443453, 2014. Disponível em: https://doi.org/10.1016/j.giq.2014.01.013. Acesso em: 20 jun. 2021.

LI, X.; PAK, C.; BI, K. X. Analysis of the development trends and innovation characteristics of Internet of Things technology - based on patentometrics and 
bibliometrics. Technology Analysis \& Strategic Management, v. 32, n. 1, p. 1-14, 2020. DOI https://doi.org/10.1080/09537325.2019.1636960.

LÓPEZ-RUBIO, P.; ROING-TIERNO, N.; MAS-TUR, A. Which regions produce themost innovation policy research?. Policy Studies, p. 1-23, 2021. DOI https://doi.org/10.1080/01442872.2021.1937595.

LOPEZ-RUBIO, P.; ROING-TIERNO, N.; MAS-VERDU, F. Assessing the Origins, Evolution and Prospects of National Innovation Systems. Journal of The Knowledge Economy, p. 1-24, 2021. Disponível em: https://link.springer.com/article/10.1007/s13132020-00712-7. Acesso em: 20 jun. 2021.

MACIAS-CHAPULA, C. A. O papel da Informetria e da cienciometria e sua perspectiva nacional e internacional. Ciência da Informação, Brasília, v. 27, n. 2, p. 134-140, maio/ago. 1998. Disponível em: http://revista.ibict.br/ciinf/article/view/794. Acesso em: 10 maio. 2021.

MASARA, B.; VAN DER POLL, J.A.; MAAZA, M. A nanotechnology-foresight perspective of South Africa. Journal of Nanoparticle Research, v. 23, n. 4, p. 1-22, 2021. Disponível em: https://link.springer.com/article/10.1007/s11051-021-05193-6. Acesso em: 20 jun. 2021.

MINGUILLO, D.; THELWALL, M. Mapping the network structure of science parks An exploratory study of cross-sector alinteractions reflected on the web. Aslib Proceedings, v. 64, n. 4, p. 332-357, 2012. Disponível em: https://arxiv.org/abs/1301.4933. Acesso em: 21 jun. 2021.

MORADI, S.; DOHKANI, F. Using the Quadruple Helix Model for evaluation of health science researches Case study of D8 countries. Library Hi Tech, v. 38, n. 4, p. 723-739, 2020. Disponível em: https://www.emerald.com/insight/content/doi/10.1108/LHT-082019-0156/full/html. Acesso em: 20 jun. 2021.

MUGNAINI, R.; CARVALHO, T.; CAMPANATTI-OSTIZ, H.. Indicadores de produção científica: uma discussão conceitual. In: POBLACION, D. A.; WITTER, G. P.; SILVA, J. F. M. Comunicação \& produção científica: contexto, indicadores e avaliação. São Paulo: Angellara, 2006. p. 313-340.

NORONHA, D. P.; MARICATO, J. de M. Estudos métricos da informação: primeiras aproximações. Encontros Bibli, v. 13, n. esp., p. 116-128, 2008. Disponível em: https://doi.org/10.5007/1518-2924.2008v13nesp1p116. Acesso em: 10 maio. 2021.

OECD. Oslo Manual 2018: guidelines for collecting, reporting and using data on innovation. 4. ed. Paris: OECD, 2018. Disponível em:

https://doi.org/10.1787/9789264304604-en. Acesso em: 15 jun. 2021.

OLIVEIRA, E. F. T. de. Estudos métricos da informação no Brasil: indicadores de produção, colaboração, impacto e visibilidade. São Paulo: Cultura Acadêmica, 2018.

PINTO, A. L. Arquivometria. ÁGORA: Arquivologia em debate, Florianópolis, v. 21, n. 42, p. 59-69, jan./jun. 2011. Disponível em:

https://agora.emnuvens.com.br/ra/article/view/263. Acesso em: 15 maio 2021. 
ROSE, R.; HOLZLE, K.; BJORK, J. More than a quarter century of Creativity and Innovation Management: the journal's characteristics, evolution, and a look ahead. Creativity and Innovation Management, v. 29, n. 1, p. 5-20, 2020. Disponível em: https://onlinelibrary.wiley.com/doi/full/10.1111/caim.12361. Acesso em: 20 jun. 2021.

SÁENZ, T. W.; GARCÍA, Emílio C. Ciência, inovação e gestão tecnológica. Brasília: CNI/IEL/SENAI/ABIPTI, 2002.

SANTOS, P. W. Q. D.; ALBUQUERQUE, J. P. S. Altmetria: uma nova lente para os estudos métricos da informação. Biblionline, v. 13, n. 3, p. 3-12, 2017. Disponível em: https://periodicos.ufpb.br/index.php/biblio/article/view/35874. Acesso em: 20 maio 2021.

SANZ-CASADO, E.; GARCÍA-ZORITA, C. Evolução dos fundamentos epistemológicos dos estudos métricos da informação. In: ENCONTRO BRASILEIRO de Bibliometria e Cientometria, 4., 2014, Recife. Anais eletrônicos [...]. 2014. Disponível em: https://www3.ufpe.br/ppgci/images/elias.pdf. Acesso em: 18 maio 2021.

SILVA, A. P. L. da; PEDROZA, F. J. A.; SOBRAL, N. V.; BUFREM, L. S. Indicadores científicos, tecnológicos e acadêmicos: uma proposta para a Univasfa partir das demandas institucionais. Informação \& Sociedade, v. 28, n. 3, p. 95-114, 2018. Disponível em: https://periodicos.ufpb.br/index.php/ies/article/view/39129.Acesso em: 20 maio. 2021.

SOUZA NETO, J.; ORLANDI, T. R. C.; SANTOS, D. L. N dos. Inovação: estratégia, gestão e cultura, 2014.

STANCATTI, D. H.; PINTO, A. L. Análise da publicação pela citação como critério de qualidade: estudo da produção científica em arquivologia. ÁGORA: Arquivologia Em Debate, v. 31, n. 62, p. 1-21, 2021. Disponível em:

https://agora.emnuvens.com.br/ra/article/view/966. Acesso em: 20 maio. 2021.

TOMAÉL, M. I.; ALCARÁ, A. R.; CHIARA, I. G. D. Das redes sociais à inovação. Ci. Inf., Brasília, v. 34, n. 2, p. 93-104, maio/ago. 2005. Disponível em:

https://doi.org/10.1590/S0100-19652005000200010. Acesso em: 05 abr. 2021.

VAN ECK, N. J.; WALTMAN, L. Vosviewer manual. Holanda: Universite it Leiden, 2018. Disponível em:

https://www.vosviewer.com/documentation/Manual_VOSviewer_1.6.8.pdf. Acesso em: 15 jun. 2021.

VANTI, N. A. P. Os links e os estudos webométricos. Ciência da Informação, Brasília, v. 34, n. 1, p.78-88, jan./abr. 2005. Disponível em:

http://revista.ibict.br/ciinf/article/view/1104. Acesso em: 10 maio. 2021.

VILELA JUNIOR, D. C. A criação de conhecimento em clusters industriais, 2010.

Tese (Doutorado em Administração) - Universidade Federal do Rio Grande do Sul, Porto Alegre, 2010.

WATANABE, C.; TSUJI, Y. S.; BROWN, C. G. Patent statistics: deciphering a 'real' versus a 'pseudo' proxy of innovation. Technovation, v. 21, n. 12, p. 783-790, 2001. DOI https://doi.org/10.1016/S0166-4972(01)00025-6 
${ }^{i}$ Mestrando do Programa de Pós-Graduação em Ciência da Informação pela Universidade Federal de Santa Catarina (UFSC). Possui Graduação em Biblioteconomia pela Universidade Federal do Amazonas (UFAM) (2019). Foi presidente do Centro Acadêmico de Biblioteconomia do Amazonas (CABAM) (2019-2020), representante discente no colegiado do curso de Biblioteconomia da Universidade Federal do Amazonas (2019-2020) e estagiário do Tribunal Regional Eleitoral do Amazonas (TRE-AM) na Seção de Biblioteca, Arquivo e Editoração (SEBIB) no período de 2018 a 2019. Atualmente, é Bolsista da Coordenação de Aperfeiçoamento de Pessoal de Nível Superior (CAPES) em nível mestrado e membro do Grupo de Pesquisa Gestão da Informação e do Conhecimento na Amazônia (GICA). Tem experiência na área de Ciência da Informação, com ênfase em estudos métricos da informação com foco na bibliometria e cientometria, ecossistemas de conhecimento e competência em informação.

ii Doutora em Avaliação de Tecnologia pela Faculdade de Ciências e Tecnologia da Universidade Nova de Lisboa (FCT-UNL). Possui graduação em Ciências Econômicas pela Universidade Regional de Blumenau (2007) e Mestrado em Economia e Gestão da Inovação pela Universidade do Porto - Portugal (2010). Atuou com consultoria em processos de inovação, gestão da inovação. Pesquisadora Associada ao Centro Interdisciplinar de Ciências Sociais da Universidade Nova de Lisboa (CICS.NOVA). Membro do Grupo de Estudos em Avaliação de Tecnologia (GrEAT). Tem-se dedicado à análise da adoção de Inovação Aberta, Avaliação de Tecnologia, Inovação Disruptiva e Inovação Tecnológica. Atualmente é Professora Adjunta no Departamento de Ciência da Informação (CIN) da Universidade Federal de Santa Catarina.

iii Pesquisador no Institute for Systems and Computer Engineering, Technology and Science (INESC TEC), Porto, Portugal. Doutor em Engenharia e Gestão Industrial pela Universidade de Aveiro, Portugal. Possui graduação em Administração pela Universidade Federal de Santa Catarina (2004), Mestrado em Gestão pela Universidade de Aveiro (2010) e MBA em Gestão para a Excelência pelo SENAI/SC (2008). Seus principais interesses de pesquisa são gestão da cadeia de suprimentos, transformação digital nas cadeias de suprimentos, gestão da inovação e gestão estratégica. Possui experiência em empresas no Brasil e em Portugal, com ênfase nas áreas de planejamento estratégico, gestão da qualidade, gestão da cadeia de suprimentos, gestão de custos e gestão de projetos.

iv Doutoranda em Arte e Design pela Universidade do Porto - Portugal. Mestre em Design com ênfase em Design Estratégico pela Universidade de Aveiro - Portugal (2010), com diploma validado pela Universidade Estadual do Rio de Janeiro - UERJ. Graduação em Design Industrial com habilitação em Projeto de Produto pela Universidade do Estado de Santa Catarina - Brasil (2005). Colabora com o Instituto de Investigação em Design, Media e Cultura - ID+ (certificado pela Fundação para a Ciência e a Tecnologia - FCT) vinculado à Universidade do Porto e à Universidade de Aveiro. Atuou como pesquisadora do Conselho Nacional de Desenvolvimento Científico e Tecnológico - CNPq junto à Universidade do Estado de Santa Catarina - UDESC, à Universidade Federal de Santa Catarina - UFSC, e ao Instituto Euvaldo Lodi de Santa Catarina desenvolvendo pesquisas nas áreas de inovação e projectos em Design. Tem experiência na área de ensino, consultoria empresarial, desenvolvimento de projetos em Design de Produto, Gestão do Design e Gestão da Inovação, e na mediação de trabalhos conjuntos entre universidades e empresas com foco na implementação de núcleos de gestão da inovação. Os interesses atuais de investigação concentram-se no desenvolvimento de estratégias de gestão da inovação para PEs baseadas no Design Estratégico com ênfase no estudo de modelos de gestão da criatividade em diferentes domínios. 